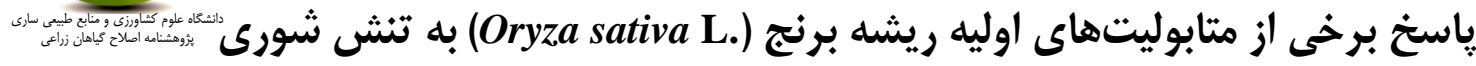

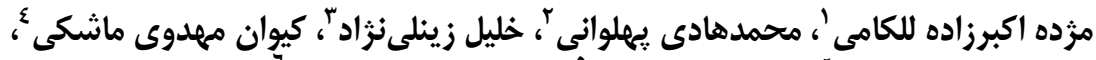

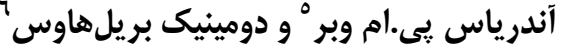

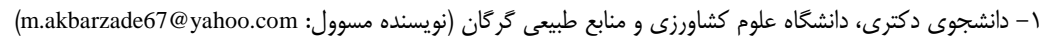

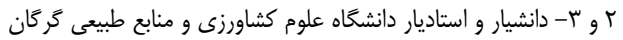

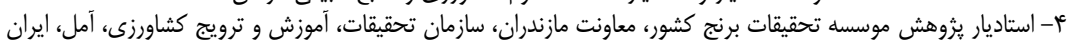

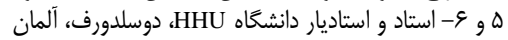

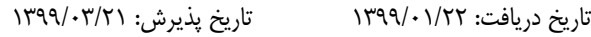

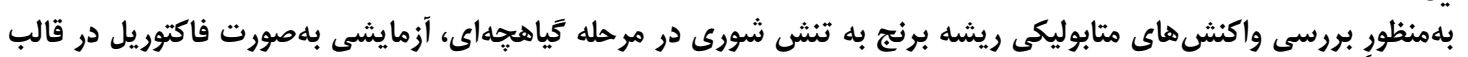

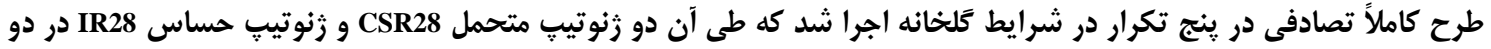

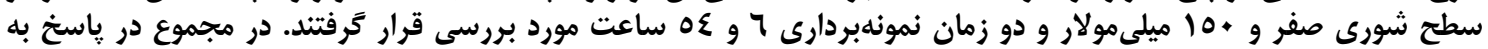

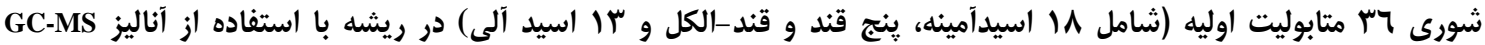

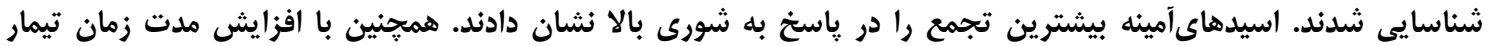

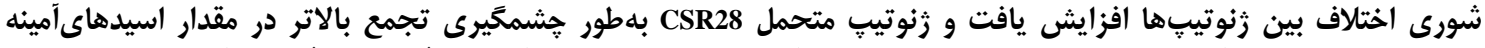

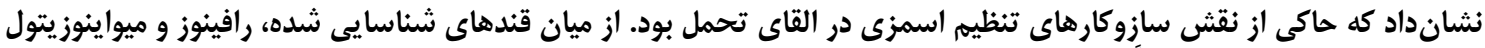

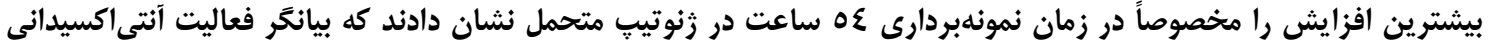

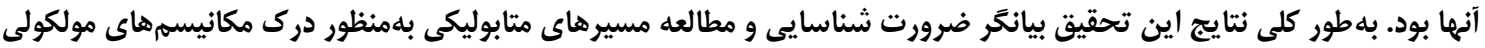

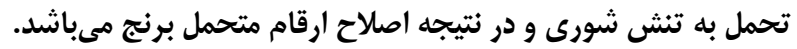

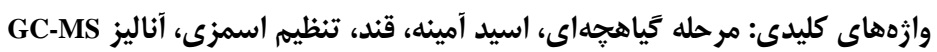

اسمزى حاصل از شورى، خشكى و سرما منجر به دهيدراته

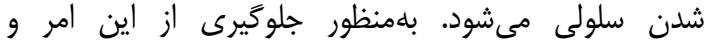

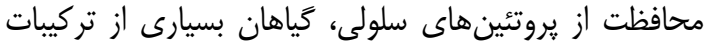

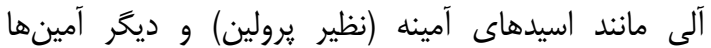

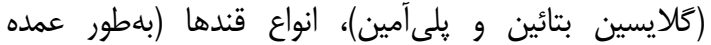

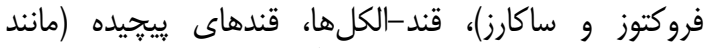
ترى هالوز و فروكتان) و اسيدهاى آلى (نظير اكزالات وات و مالات

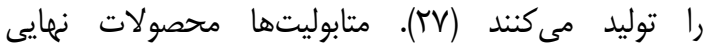

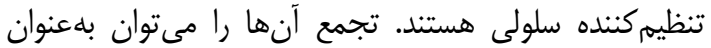

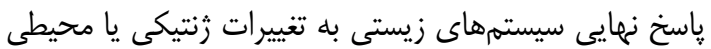

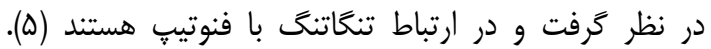

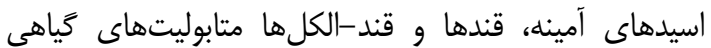

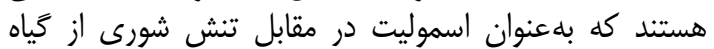

محافظت مى كنند (آ).

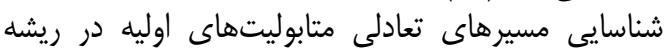

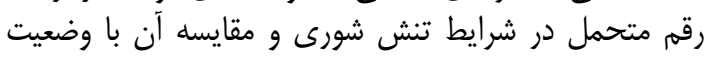

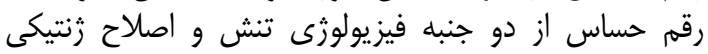

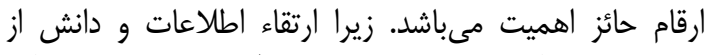

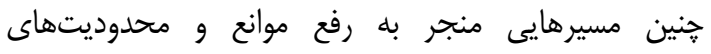

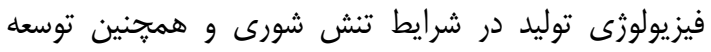

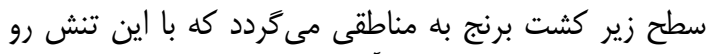

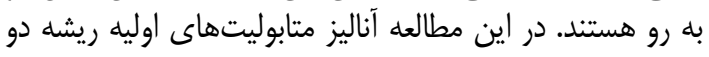

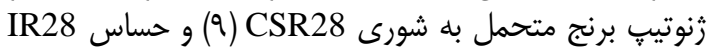

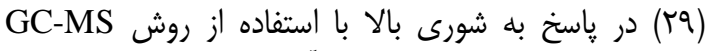

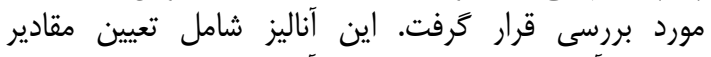

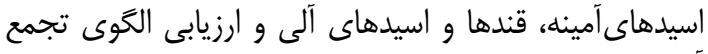

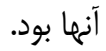

مقدمه

تنش شورى يكى از مهمترين تنشهاى محيطى است كه

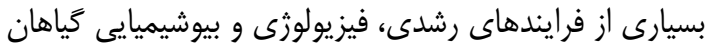

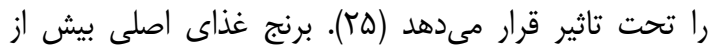

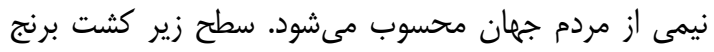

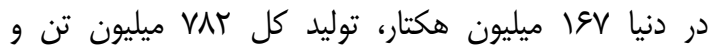
عملكرد متوسط \&V/

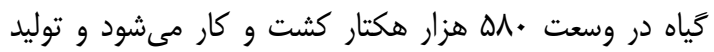

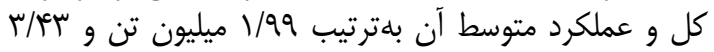

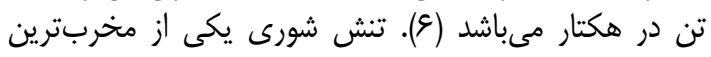

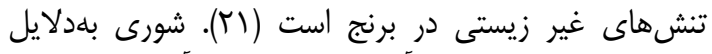

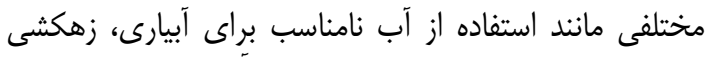

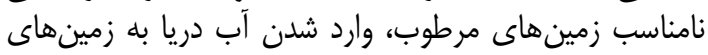

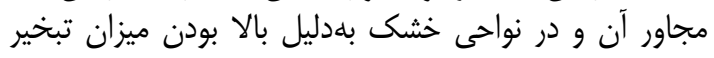

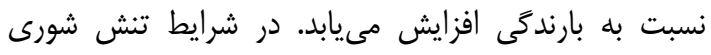

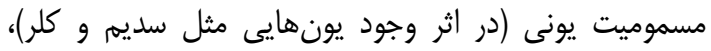

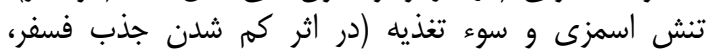

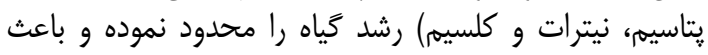

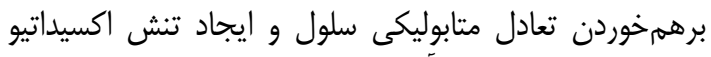

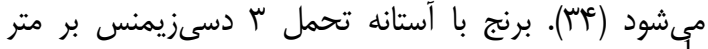

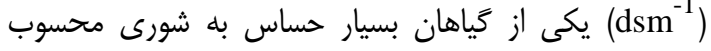

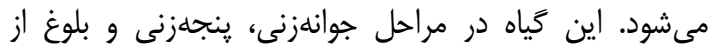

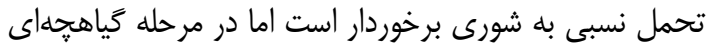

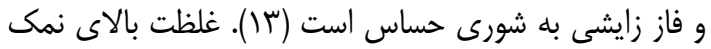

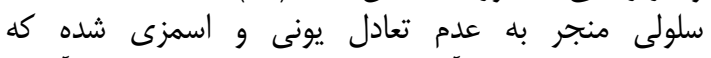

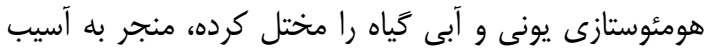

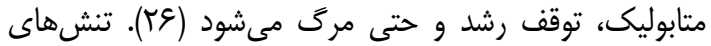


ابتدا نمونهها بلمدات • ب دقيقه در

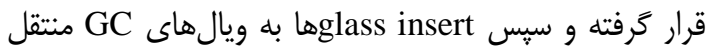

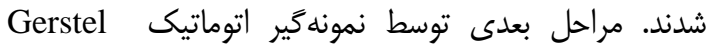

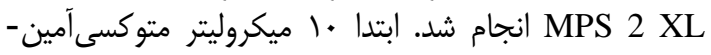

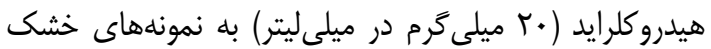

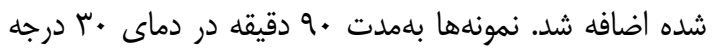

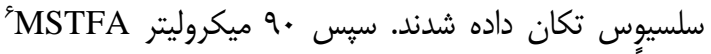

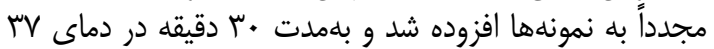

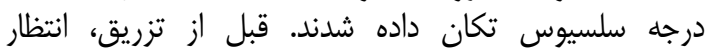

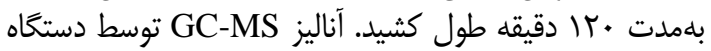
و آناليز دادهها نيز توسط النيط (Agilent) 7200 GC-QTOF كوي (Agilent) Mass Hunter Software

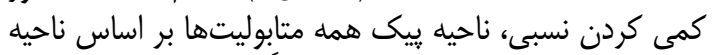

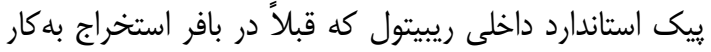

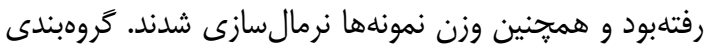

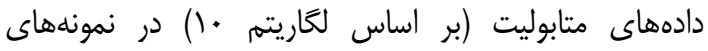
MeV v4.9.0 مختلف توسط رسم نقشه دمايى در نرمافزار

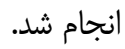
آناليز آمارى دادهامها دادها دادههاى حاصل بهصورت دادها فاكتوريل در قالب طادرح كاملاً

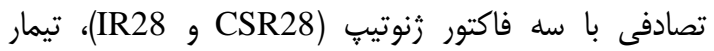

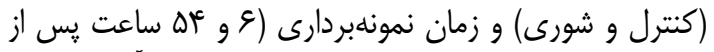

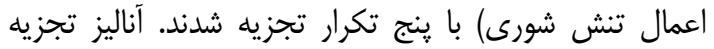
واريانس توسط نرمافزار

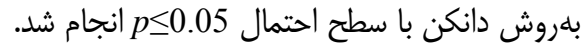

\section{نتايج و بحث}

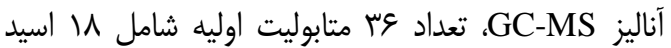

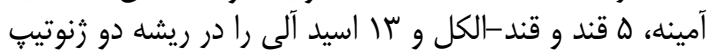

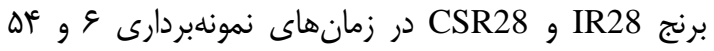

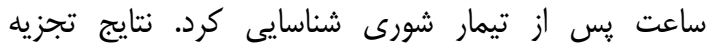

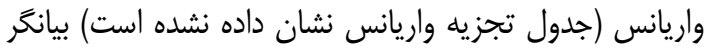
اختلاف معنىدار بين شرايط كتترل و تنش برائ براي وس مت متابوليت

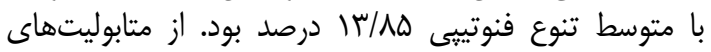

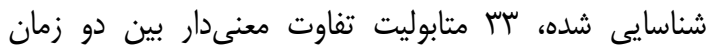

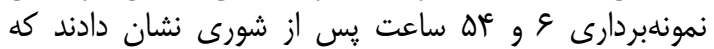

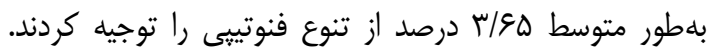

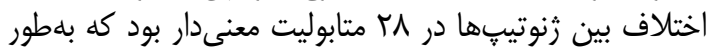

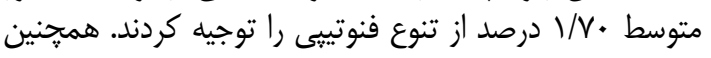

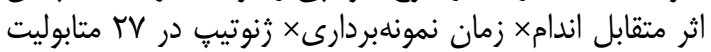

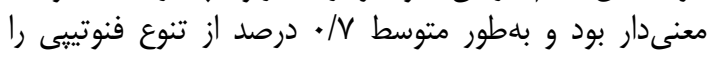
توجيه كرد.

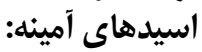

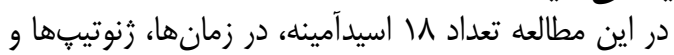

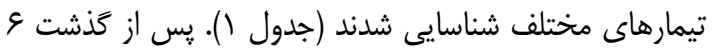

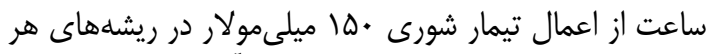

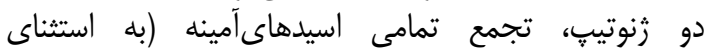

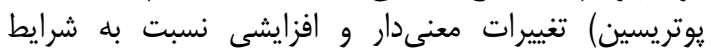

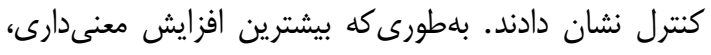

\section{مواد و روش هاد مواد كياهى و اعمال تيمار تنش شورى}

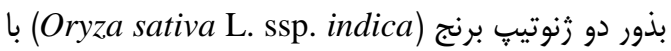

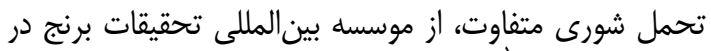
فيلييين (IRRI) تهيه شوريه شدند. زنوتيه (IR833-6-2-1-1/IR1561-149-1//IR24*4/O. nivara

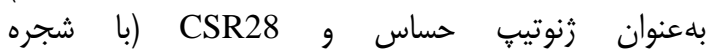

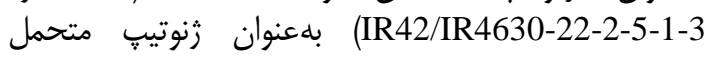
بلترتيب در IRRI و موسسه تحقيقات شورى خاى در هند ' (CSSRI)

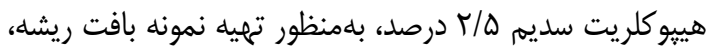

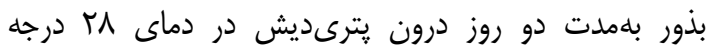

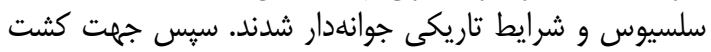

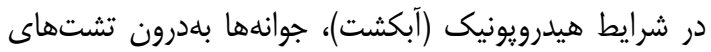

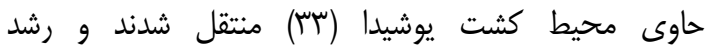

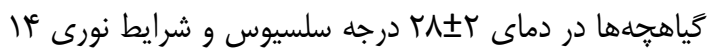

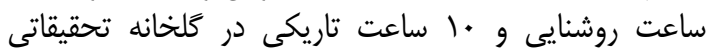

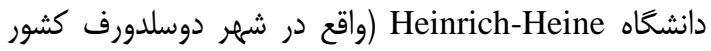

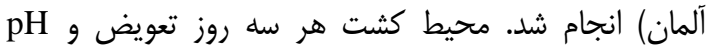

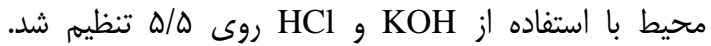

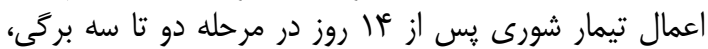

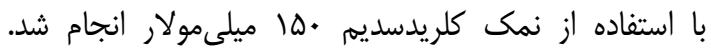

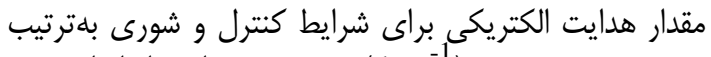

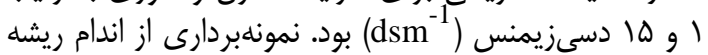

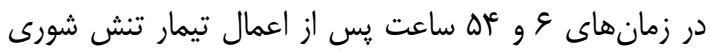

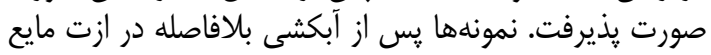

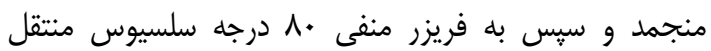

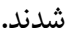

استخراج و اندازهكيرى متابوليتها

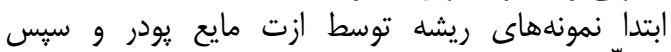

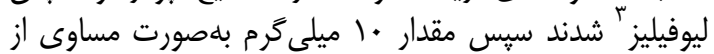

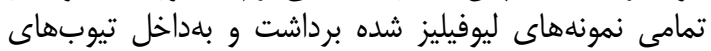

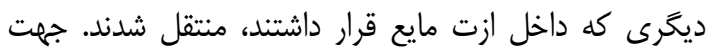

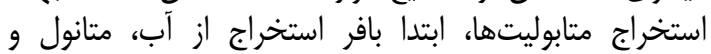

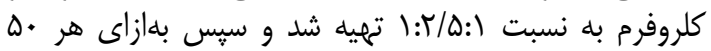

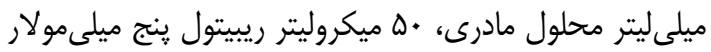

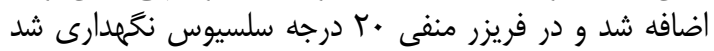

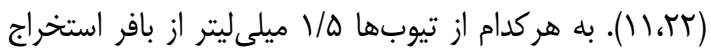

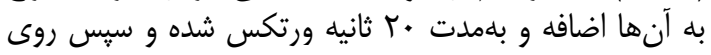

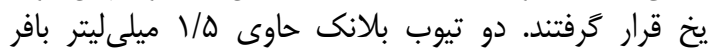

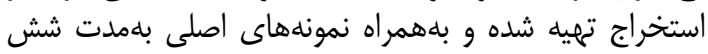

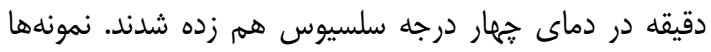

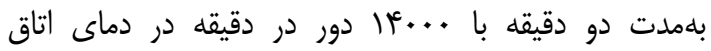

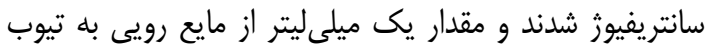

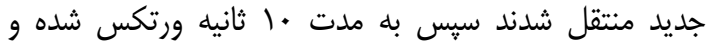

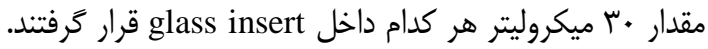

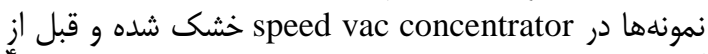

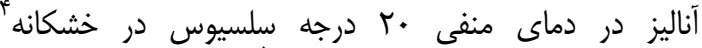

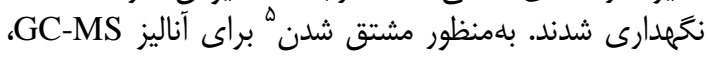

1- International Rice Research Institute

4- Desiccator

$\mathrm{N}$-trimethylsilyltrifluoroacetamide
2- Central Soil Salinity Research Institute 5- Derivatization
3- Lyophilized 6- N-methyl- 
نتايج با يثوهشهاى ديخر در زمينه نقش اسيدهاى آمينه در

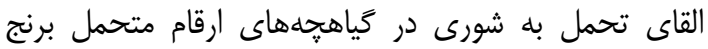

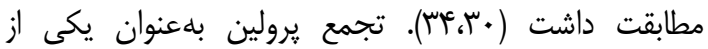

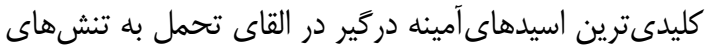

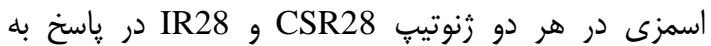
شورى بالا افزايش يافت. تجمع مقادير زياد يرولين يكى رياسخ

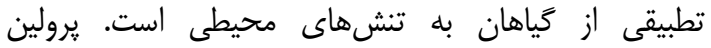

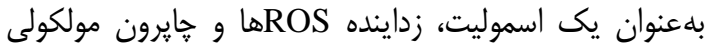

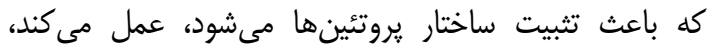

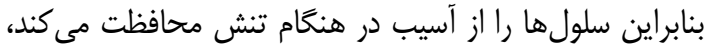

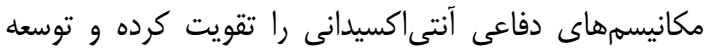

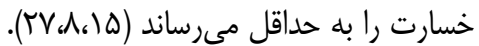

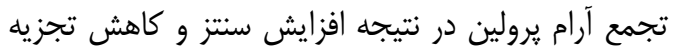

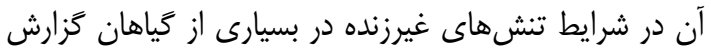

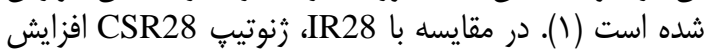
ديرتر و طولانى تر در اسيدهاى آمينه نشان داد كه اين اين نتايج با

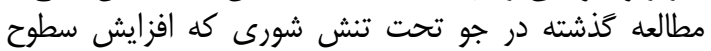

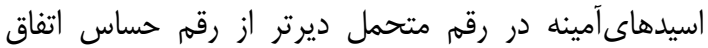

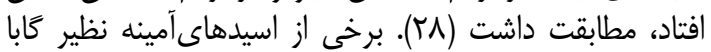

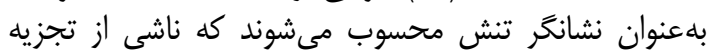

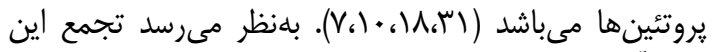

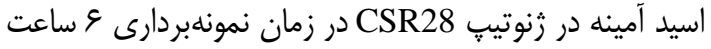

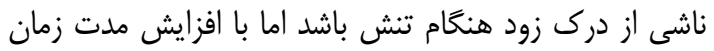

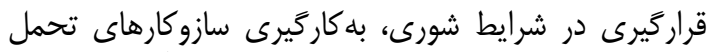

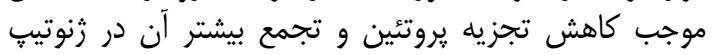
متحمل شدج.
مربوط به اسيدآمينه ايزولوسين در ريشههاى CSR28 و IR28

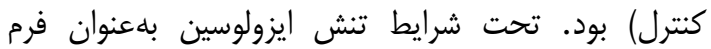

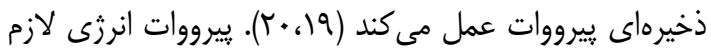

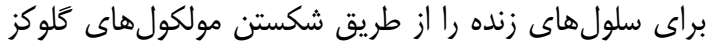

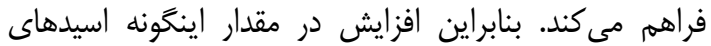

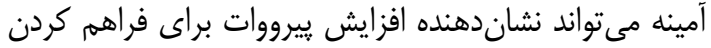

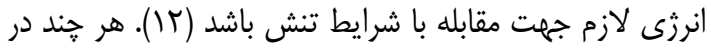

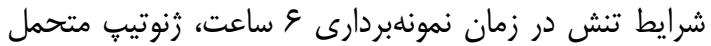
(CSR28)

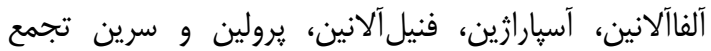

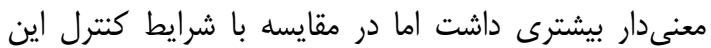

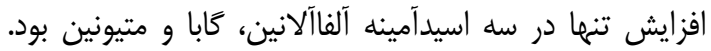

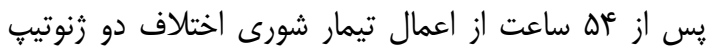

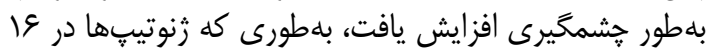

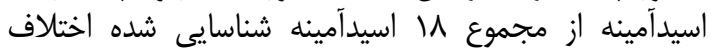

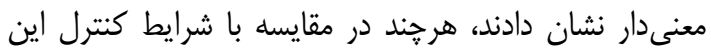

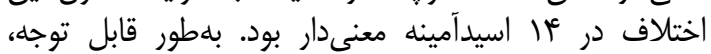

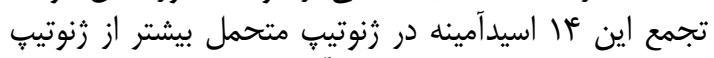

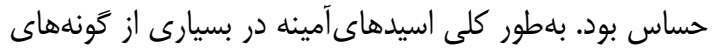

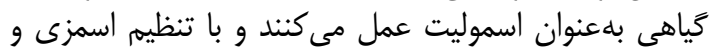

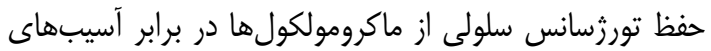

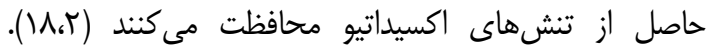

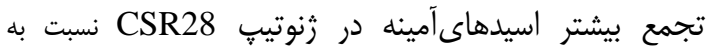

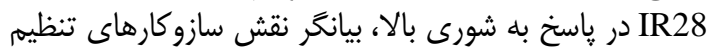

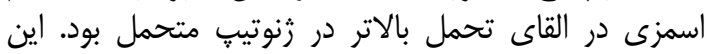

جدول ا- مقايسه ميانگين اسيدهاى آمينه در دو زنوتيت متحمل و حساس برنج، تيمارها و زمانهاى نمونهبردارى مختلف با استفاده از آزمون دانكن $(P \leq 0.05)$

Table 1. Mean comparison of amino acids of the two tolerant and sensitive rice genotypes, the treatments and the time points using Duncan' test. Common letters in each row indicate non-significant differences

\begin{tabular}{|c|c|c|c|c|c|c|c|c|}
\hline \multicolumn{4}{|c|}{ شورى } & \multicolumn{4}{|c|}{ كنترل } & \multirow{3}{*}{ اسيدهاى آمينه } \\
\hline \multicolumn{2}{|c|}{ ساعت DF } & \multicolumn{2}{|c|}{ و ساعت } & \multicolumn{2}{|c|}{ ل ساعت DF } & \multicolumn{2}{|c|}{ ع ساعت } & \\
\hline IR28 & CSR28 & IR28 & CSR28 & IR28 & CSR28 & IR28 & CSR28 & \\
\hline $911 / 7 \pm 11 / .9^{d}$ & $|r \Lambda| / T \pm \mid r T / \Delta^{c}$ & $r r \& q / q \pm 1 r N q^{b}$ & $\Pi \backslash \wedge V / \varepsilon \pm \| r \backslash /\left.\right|^{a}$ & $\| \& r / / \pm r \mid / \wedge^{\mathrm{cd}}$ & $\| \varepsilon V / r \pm F r / q^{c d}$ & $|r \wedge| / r \pm q \cdot / \kappa^{c}$ & $\mid f \cdot q / r \pm r \cdot / \cdot r^{c}$ & آلفاآلانين \\
\hline$\Delta r \cdot q / \backslash \pm r q \cdot / v^{d}$ & $\mid r r \cdot N a \pm r \cdot r / r^{a}$ & $\vee \backslash \& q / \Lambda \pm \mid f y / \cdot \Lambda^{c}$ & $\wedge V \backslash \varepsilon / \varepsilon \pm V V^{c} / \Lambda^{b}$ & $|Q \Gamma F / Q \pm \|| \mid r / r^{f}$ & $|f| r / f \pm \mid f q / \Delta^{f}$ & $g \cdot r / r \pm 1 r / r^{g}$ & $r \wedge . . / q \pm \mid r g / \mu^{\mathrm{e}}$ & آسياراراثين \\
\hline $1 \cdot \Lambda \Delta r / r \pm I V{ }^{c} / q^{d}$ & $19 \& 9 V / \triangle \pm r \mid r / q^{b}$ & $r r q V r / \Delta \pm \Delta \cdot r /\left.r^{a}\right|^{a}$ & $|V \times q q / V \pm| q V / \cdot r^{c}$ & $\vee \varepsilon \wedge r / १ \pm r \cdot \varphi / \varsigma^{f}$ & $v \cdot v r / \Lambda \pm r \cdot q / \cdot r^{f}$ & $q \Lambda \| F / q \pm r F \cdot / \cdot r^{e}$ & 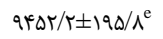 & آسيارتات \\
\hline$\Lambda) / \Delta \pm \Delta / q^{c}$ & $r \Delta \backslash / \backslash \pm 1 q / \cdot \Delta^{a}$ & $r \Delta N \wedge \pm \Lambda^{a}$ & $r \cdot r / v \pm 1 \Delta / r^{b}$ & $r \varepsilon / r \pm r / r^{d}$ & $r r / T \pm 1 / q^{d}$ & $s r / q \pm F / r^{c}$ & $V Q / r \pm r / \zeta^{c}$ & بتاآلانين \\
\hline $\mid \Delta r / q \pm V / \Delta^{c}$ & $r \Delta \Delta / \wedge \pm 1 \Delta / \Delta^{c}$ & $|r \vee \Delta / v \pm| \Lambda r / q^{a b}$ & $1 \cdot r \varepsilon / r \pm 11 / \cdot r^{b}$ & $r \mid N q \pm r / v^{c}$ & $11 \omega \cdot / \Delta \pm \Delta V / \mu^{a b}$ & $\mid r \varepsilon \cdot / \kappa \pm v \varepsilon / q^{a}$ & $r V V / \cdot r \pm I \Delta / \cdot \wedge^{c}$ & كابا \\
\hline$r \mu r / \Lambda \pm \mid r / \% \mu^{d d}$ & $V \cdot r / r \pm r \mid / r^{a}$ & $\varepsilon \wedge \kappa / r \pm \kappa q / r^{a}$ & $q \mu r / \Delta \pm q / r^{a}$ & $r \Delta \backslash / \backslash \pm \mid r / r^{e}$ & $r \& 9 / q \pm 11 / \Gamma^{\mathrm{de}}$ & $r \varepsilon \Delta / \& \pm r \cdot / \kappa^{c}$ & $\Delta r \Delta / r^{w} \mid \cdot / \kappa^{b}$ & كَايسين \\
\hline $1 \cdot \wedge \cdots / v \pm r \mid r / q^{d}$ & $\mid r r V c / \Lambda \pm 19 \Delta / q^{c}$ & $198 \times T / \Delta \pm r Y \& / V^{a}$ & $|V \wedge \mu| \pm \mid f \Psi / \cdot V^{b}$ & $q r q \vee / \Delta \pm r r \Delta / \cdot \Delta^{e}$ & 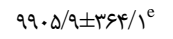 & $\| \Delta Q \Delta / \wedge \pm \mu \mid \mu / /^{c d}$ & $\|\varepsilon \Delta\| / r \pm q r / \wedge^{c}$ & كلوتامات \\
\hline$r \Lambda / / q \pm r N \xi^{d}$ & $q \vee r / \Psi \pm \varepsilon / \Delta^{c}$ & $\mid r \varepsilon / / \Lambda \pm r \cdot / \leftarrow^{a}$ & $1.9 N \Delta \pm r Y /^{b}$ & $\wedge \vee / \wedge \pm \Delta^{\mathrm{e}}$ & $\Delta r / q \pm F / /^{e}$ & $\Delta r / r \pm r / \Lambda^{e}$ & $c \cdot / \Delta \pm r / Q \Lambda^{e}$ & ايزولوسين \\
\hline$r \in V / \Lambda \pm 1 \cdot / \Lambda^{d}$ & $r \mid Q / A \pm r r / \digamma^{c}$ & 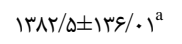 & $\Lambda \Delta F / \cdot r \pm q / \Lambda^{b}$ & $\Lambda T / r \pm 1 / \wedge^{e}$ & $\kappa r / r \pm r / q^{e}$ & $\Delta N \Delta \pm \Delta / r^{\mathrm{e}}$ & $q q / . q \pm \mu / \mu^{\mathrm{e}}$ & ل ل لوسين \\
\hline$\Gamma / r / r \pm V / \Lambda^{c}$ & $1 . r q / g \pm 1 r g / v^{b}$ & $\| \wedge \mu \pm \kappa r / \Delta^{a}$ & 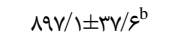 & $1 . q / \varepsilon \pm r / q^{d}$ & $q \tau / \Delta \pm r / \kappa^{d}$ & $\mid r r / \varepsilon \pm \psi / \gamma^{d}$ & $11 \cdot / \kappa \pm r /\left.\right|^{d}$ & ليزين \\
\hline$r r q / \cdot r \pm|\varepsilon /|^{c}$ & $\Delta \wedge V / \wedge \pm \Delta \mathrm{Q} / \wedge^{\mathrm{a}}$ & $r q^{c} v / v \pm 11 / \kappa^{b}$ & $<q 4 / \varepsilon \pm 11 / \Lambda^{b}$ & $\| \varepsilon / \varepsilon \pm \psi / \mu^{d}$ & $v Q / r \pm \Delta / r^{d}$ & $\varepsilon s / r \pm \kappa / q^{d}$ & $\kappa q / r \pm \Delta / r^{d}$ & متيونين \\
\hline$\Delta F V / . \& \pm N / V^{d}$ & $\wedge ৭ \& \pm 1 \cdot / \wedge^{\mathrm{a}}$ & $9 \varepsilon \cdot / r \pm r \cdot / \Lambda^{c}$ & $\wedge 1 q / v \pm 1 v / a^{b}$ & $111 / v \pm 1 / \cdot r^{f}$ & $\| 1 / / \leftarrow \pm 1 / r^{f}$ & $\mid r v / f \pm \varepsilon / \mu^{f}$ & $r F \cdot / q \pm 1 . / 1^{e}$ & فنيل آلانين \\
\hline$\Gamma \wedge r / \Delta \pm r \cdot / r^{a}$ & $\Delta r r / g \pm r \cdot / r^{a}$ & $\| r V \pm N \Lambda^{c}$ & $194 / \Delta \pm 11 / v^{b}$ & $r \varphi / \cdot \kappa \pm r / r^{d}$ & $\tau \cdot / \Delta \pm \cdot / q^{\mathrm{d}}$ & r & $\kappa r / \Lambda \pm r / r^{d}$ & يرولين \\
\hline$r r v / v \pm 19 / \gamma^{e}$ & $\Lambda N \& \pm \uparrow r / \Lambda^{\mathrm{d}}$ & $1994 / \Delta \pm 1 N / v^{b}$ & $\mid f 4 N / q \pm r \Delta / q^{c}$ & $V V V / \Delta \pm r \mid / r^{d}$ & $\mid Q T G / G \pm q T / V^{c}$ & $19 \wedge \mathrm{a} / \Delta \pm \Delta 1 / \kappa^{\mathrm{b}}$ & $r q \| V / r \pm r r / \Delta^{a}$ & يوتريسين \\
\hline$\Delta \omega F \backslash / r \pm \Gamma \Delta \Gamma / \mathcal{Y}^{c}$ & $1 . \mu \backslash \Delta / q \pm r F q / \Delta^{b}$ & $1 \cdot \Delta r / / \kappa^{a} \pm r \cdot \Delta / r^{b}$ & $|r| \cdot r / \Lambda \pm r q r / q^{a}$ & $\mid f \vee \Delta / r \pm \wedge q / \cdot \kappa^{e}$ & $\mid V \in \mathcal{E} / \mathcal{F} \pm r r / \kappa^{e}$ & $|r \mu r / \Lambda \pm g| / r^{\mathrm{e}}$ & 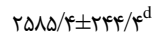 & سرين \\
\hline $1 \% V \Delta / \Delta \pm 1 \cdot 1 / q^{c}$ & $19 \cdot r / q \pm V V / \Delta^{a}$ & $1 \varepsilon \cdot 1 / 1 \pm q \cdot / \mu^{b}$ & $|\varepsilon \cdot N / r \pm \|| F^{\prime} / \xi^{b}$ & $r q \varepsilon / \& \pm r N q^{d}$ & $r \in \varepsilon / \Lambda \pm \mid r / \kappa^{d}$ & $r r \mid r \pm I r / V^{d}$ & $r V q / r \pm W N e^{d}$ & ترئونين \\
\hline$\Delta V \cdot / r \pm r q / \Lambda^{e}$ & $\| r / / \& \pm \varnothing \varepsilon / \Lambda^{c}$ & $\mid \Delta \varsigma \varnothing / \mu \pm \kappa \kappa / \cdot \Lambda^{b}$ & $r \cdot \cdot r / r \pm q / \cdot \omega^{a}$ & $f v g / V \pm r / / V^{f}$ & $r \wedge q / f \pm 1 q / q^{f}$ & $r V T / V \pm I r / . r e f$ & $V \| r / s \pm r r / \Lambda^{d}$ & تيروزين \\
\hline$v q v / Q \pm r N q^{d}$ & TrTNa $\pm 1 Q F / I^{\mathrm{b}}$ & & $\mid \Lambda V G / ৭ \pm r N / \mu^{c}$ & $r \Delta s / q \pm r / / \Lambda^{e}$ & $|N N r \pm| r / \cdot \Lambda^{\mathrm{e}}$ & $r V T / V \pm T E / T^{e}$ & rergg $\pm 1 r / K^{e}$ & والين \\
\hline
\end{tabular}


در ياسخ به شورى كاهش يافت. قندها علاوه بر منابع

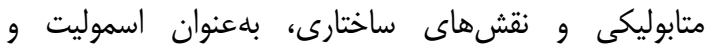

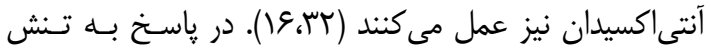

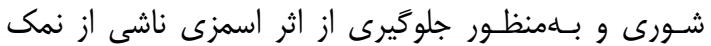

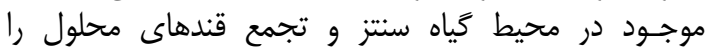

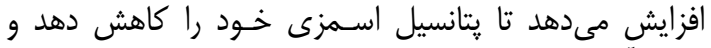

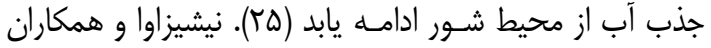

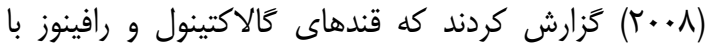

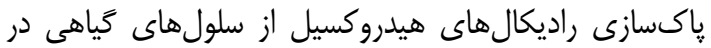

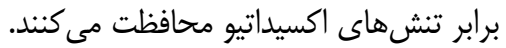

قندها و قند-الكلها: آناليز GC-MS منجر به شناسايى ينج

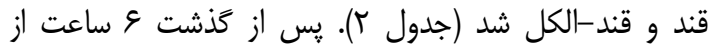

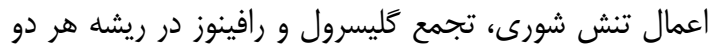

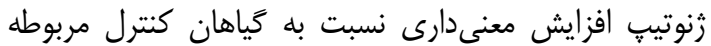

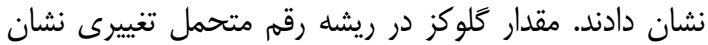

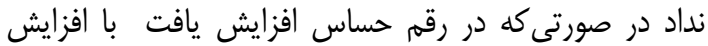

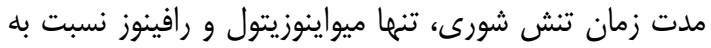

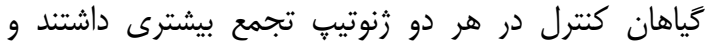

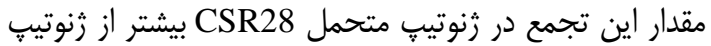

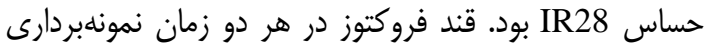

جدول r- مقايسه ميانكين قندها و قند-الكلها دو زنوتيقٍ متحمل و حساس برنج، تيمارها و زمانهاى نمونهبردارى مختلف با استفاده از آز آزمون دانكن (P

Table 2. Mean comparison of sugars and sugar alcohols of the two tolerant and sensitive rice genotypes, the treatments and the time points using Duncan' test

\begin{tabular}{|c|c|c|c|c|c|c|c|c|}
\hline \multicolumn{4}{|c|}{ شورى } & \multicolumn{4}{|c|}{ كنترل } & \multirow{3}{*}{ قندها، قند- } \\
\hline \multicolumn{2}{|c|}{ ماع هاعت } & \multicolumn{2}{|c|}{ צ ساعت } & \multicolumn{2}{|c|}{ } & \multicolumn{2}{|c|}{ צ ساعت } & \\
\hline IR28 & CSR28 & IR28 & CSR28 & IR28 & CSR28 & IR28 & CSR28 & \\
\hline $9 \wedge 9 / r \pm 19 / /^{\mathrm{se}}$ & $\mu \varphi g / \Delta \pm \Gamma V / \Gamma^{f}$ & $|r| r / r \pm F V / q^{c}$ & $1.9 \Psi / I \pm r r / r^{d}$ & $|\Pi| \Delta / \wedge \pm \Delta \Gamma / /^{b}$ & $\mid \Lambda F r / \Delta \pm r g / \Lambda^{a}$ & $\Delta f g / \lambda \pm r r / V^{f}$ & $1 \cdot \Gamma N / \Delta \pm \Delta / r^{d}$ & كلوكز \\
\hline 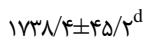 & $\mid f \cdot V / r \pm Y N / r^{e}$ & $r \vee \varepsilon \Delta / \Delta r \pm r \cdot{ }^{a}$ & $r r \Delta V / \Delta \pm V I / \kappa^{b}$ & $r \mu r q / r \pm q \varepsilon / r^{b}$ & 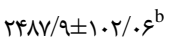 & 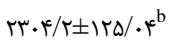 & $r \ldots / \Lambda \pm \Delta r / r^{c}$ & كليسرول \\
\hline $191 \varepsilon / \varepsilon \pm r V v / \Delta^{f}$ & $1 \cdot 1 s / 1 \pm v g / v^{\mathrm{g}}$ & $\Pi 19 \Delta / \Delta \pm \varepsilon \Delta / \Lambda^{e}$ & $|\varepsilon r V / / \pm| q \mid / r^{\mathrm{d}}$ & $1 r \cdot v \Delta / \Lambda \pm g \Delta f / \Lambda^{a}$ & $11 \cdot \wedge \varepsilon / \wedge \pm V / \Lambda^{b}$ & $\Delta r \wedge Q / \Delta \pm \mid \xi N / V^{\mathrm{d}}$ & $8190 / \wedge \pm r \cdot 1 / /^{* c}$ & فروكتوز \\
\hline$\| V q / \perp \pm \Delta v / \kappa^{d}$ & $r \Delta r q / \Delta \pm \Delta N / r^{a}$ & $\| \varepsilon \Psi / q \pm N / F^{d}$ & $10 \& V \pm 1 \cdot / \kappa^{b}$ & $৭ \Delta Q / \cdot \Lambda \pm r r / \Lambda^{e}$ & $|f| r / V \pm \mid r / \cdot \Delta^{c}$ & $\| F N / \varepsilon \pm 1 r / \kappa^{d}$ & $|\Delta| r / r \pm r \cdot /^{b}$ & ميواينوزيتول \\
\hline$\Gamma^{\prime} \Delta / v \pm I f / \Gamma^{b}$ & $r q r / \kappa \pm 1 r / \kappa^{c}$ & & Tr/a $\pm 1 / \Lambda^{b}$ & $N r_{ \pm} \cdot / \kappa^{d}$ & $s / s \pm \cdot / \kappa^{\mathrm{d}}$ & $1 \omega / \psi^{ \pm} \cdot / \kappa^{\mathrm{d}}$ & $W / \mu^{\prime} \pm \cdot /^{\mathrm{d}}$ & رافينوز \\
\hline
\end{tabular}

مقايسه با IR28 (زنوتيب حساس)، در زنوتي؟

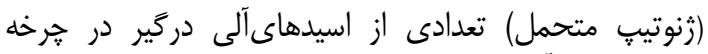

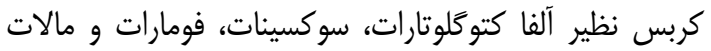

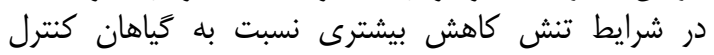

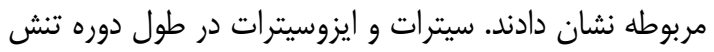

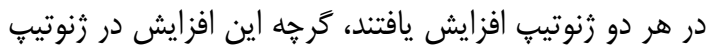

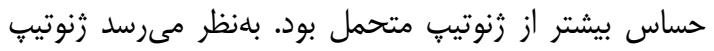

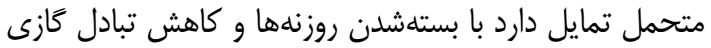

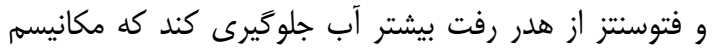

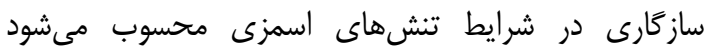

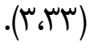

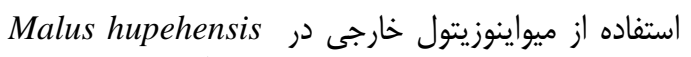
Rehd

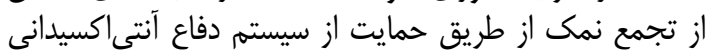

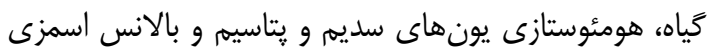
شد (1) (1). اسيدهاى آلى: در يثوهش حاضر سا اسيد آلى در ريشه گياه

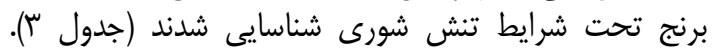

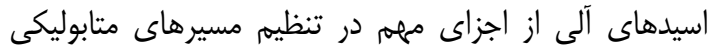

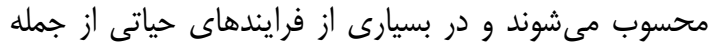

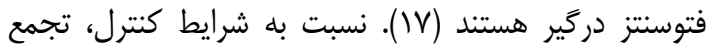

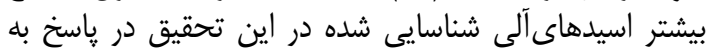

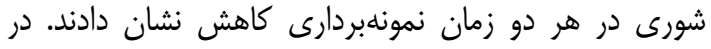


جدول س- مقايسه ميانگ̌ين اسيدهاى آلى دو زنوتيٍٍ متحمل و حساس برنج، تيمارها و زمانهاى نمونهبردارى مختلف با استفاده از آزمون دانكن (P 10.05$)$

Table 3. Mean comparison of organic acids of the two tolerant and sensitive rice genotypes, the treatments and the time points using Duncan' test

\begin{tabular}{|c|c|c|c|c|c|c|c|c|}
\hline \multicolumn{4}{|c|}{ شورى } & \multicolumn{4}{|c|}{ كنترل } & \multirow{3}{*}{ اسيدهاى آلى } \\
\hline \multicolumn{2}{|c|}{ 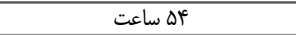 } & \multicolumn{2}{|c|}{ عاعت } & \multicolumn{2}{|c|}{ 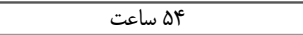 } & \multicolumn{2}{|c|}{ عاعت } & \\
\hline IR28 & CSR28 & IR28 & CSR28 & IR28 & CSR28 & IR28 & CSR28 & \\
\hline $\mathrm{T} / \mathrm{g} \pm \mathrm{I} / \mathrm{V}^{g}$ & $r q / q \pm r / /^{g}$ & $1 q / / q \pm 1 / \phi^{1}$ & $V a / v \pm 1 / \Delta^{a}$ & $\Delta V / \Delta \pm r / \cdots V^{e}$ & $1 \cdot 1 / 9 \pm \cdot / V^{0}$ & $91 / 1 \pm 4 / c$ & $|V| V \pm f / r^{a}$ & آلفاكتو گلوتارات \\
\hline$\Delta r V * / / \pm r r / \gamma^{c}$ & $r \cdot r|/ V \pm| Q\rangle / \mu^{e}$ & $v q \cdot N \mathcal{F} \pm r m r / q^{a}$ & $\Delta M M \cdot / V \pm I f V / r^{b}$ & $g \widetilde{q} / r \pm r r / r^{f}$ & $\Lambda v \cdot \pm r \cdot / \gamma^{f}$ & $\psi M \Lambda \Delta / r \pm 1 \cdot \psi / \Lambda^{d}$ & $\mid q 1 \cdot / \pm 11 \cdot / \xi^{\mathrm{d}}$ & سيترات سايزو \\
\hline $1 \% \cdot / \Delta \pm N q^{a}$ & $\| 1 f / \mu \pm \varepsilon / \Lambda^{a}$ & $\mid r \cdot / v \pm N \cdot . r^{a}$ & $\mid r \varepsilon / r \pm N v^{a}$ & $r r \Lambda \pm r / q^{c}$ & $r \Lambda 1 / ৭ \pm r \cdot / \Delta^{D}$ & $r r \varepsilon / q \pm r / q^{a}$ & $r \Delta V / q \pm 1 . / q^{D}$ & فومارات \\
\hline$V \varphi / r \pm r / \cdot r^{D}$ & $\Lambda v / \Delta \pm r / \Lambda^{a}$ & $q) / \backslash \pm r / r^{a}$ & $\Lambda q / r \pm r / \Delta^{a}$ & $\checkmark r / \Lambda \Lambda \pm r / \Lambda^{0}$ & $V / / \varepsilon \pm 1 / r^{D}$ & $\varepsilon s / \mathrm{V} \pm \varepsilon / \Lambda^{0}$ & $v \psi / \Delta \pm \tau / \gamma^{D}$ & كليسرات \\
\hline$s \varepsilon / V \pm r / \cdot \mu^{\mathrm{d}}$ & $|f \uparrow / V \pm \|| / \varsigma^{c}$ & $|f| / q \pm \mid r / \Delta^{c}$ & $10 \cdot / \mu \pm \mu / \Delta^{c}$ & $\| V / \mu \pm \psi / \mu^{c}$ & $\mid r \varepsilon / \Lambda \pm r / q^{c}$ & $r \cdot \varepsilon / \Delta \pm \Delta / \Lambda^{\mathrm{b}}$ & $r g r / r \pm r V / v^{a}$ & كليكولات \\
\hline$\kappa \cdot / \Lambda \pm 1 / r^{\mathrm{a}}$ & $৭ \varepsilon / \cdot \wedge \pm \cdot / \mu^{D}$ & $s / / \Delta \pm r / /^{c}$ & $|\xi \kappa / \& \pm| r / \Delta^{a}$ & $s \cdot / \xi \pm r / r^{c}$ & $q / / 9 \pm r / r^{c}$ & $r \varepsilon / T \pm 1 / r^{c a}$ & $\Gamma / \Lambda \pm r / \mu^{\alpha}$ & هيدروكسى كلوتارات \\
\hline $111 / q \pm V / q^{a}$ & $q . / ৭ \pm \varnothing / r^{c}$ & $1 \cdot V / r \pm \tau / \gamma^{\mathrm{ab}}$ & $1 \cdot r / \& \pm \cdot / \Delta^{a b}$ & $r V / \Lambda \pm / / \wedge^{a}$ & $r \Delta / \Delta \pm \cdot / v^{a}$ & $\Lambda ه / \Lambda \pm r / q^{c}$ & $৭ \& / \wedge \pm 1 / \wedge^{\mathrm{Dc}}$ & ايزوسيترات \\
\hline$V^{\mu}+/ \Delta \pm 1 \Delta / /^{D}$ & $V<r / q \pm r q / \xi^{D}$ & $11 \Delta \cdot / \Delta \pm \mid \Lambda V / \cdot \mu^{\mathrm{a}}$ & $1 \cdot \Lambda \Delta / r \pm I r r / r^{a}$ & $V q \& / r \pm 1 \omega / \digamma^{D}$ & $V \wedge r / \Delta \pm r Y / V^{D}$ & $11 q 4 / \cdot \Lambda \pm r q / r^{a}$ & $1 \cdot \Delta r / q \pm r r / r^{a}$ & لاكتات \\
\hline $\mid V G N \mu \pm V \varepsilon / \Lambda^{t}$ & $1190 / \Lambda \pm \Lambda \varepsilon / \mu \mathrm{g}$ & 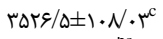 & $r \Delta \mid V / \cdot r \pm \Phi g / \Delta^{c}$ & $r \mid r \Delta / v \pm \Delta \Delta / \mu^{\mathrm{e}}$ & $M 1 q \cdot \pm q \cdot / \wedge^{d}$ & $\varphi 998 / \Lambda \pm I I V / \wedge^{D}$ & $s<q r / \wedge \pm r v / . \varphi^{a}$ & مالات \\
\hline$r / / \Psi \pm r / \mu^{e}$ & $r N / T \pm 1 / v^{e}$ & $q r / ৭ \pm r / \wedge^{b c}$ & $\varepsilon r / s \pm 1 / 1^{\text {bcd }}$ & $\Delta s / \wedge \pm \Gamma / \Delta^{c d}$ & $\Delta F / \cdot \lambda \pm 1 / \Lambda^{a}$ & $\varepsilon N V \pm \triangle / \mathcal{F}^{\mathrm{b}}$ & $V N \backslash \pm r / \cdot \wedge^{a}$ & ي ييرووات \\
\hline$q \Lambda r \pm r q / \Delta^{t}$ & TETr/I $\pm 1 \cdot r / g^{c}$ & $\mid \varepsilon r \cdot / r \pm \Lambda V / \Delta^{\mathrm{de}}$ & $r \xi q s / r \pm 1 \varepsilon \cdot / \Lambda^{\mathrm{D}}$ & $1 r \Delta q / r \pm \Delta r / \varsigma^{e}$ & $r m q r / q \pm \mid N /^{c}$ & $\mid \wedge \mu r / \varepsilon \pm \Delta \psi / \cdot^{\mathrm{d}}$ & $r V \gamma^{c} \cdot / \pm I r V / g^{a}$ & كوئينات \\
\hline$\Delta V \Delta / \Delta \pm r v / \kappa^{I}$ & $\| \varepsilon V / r \pm r r / V^{c}$ & $999 / 1 \pm 10 / r^{e}$ & $\mid \leftarrow \wedge r / \wedge \pm r r / v^{a}$ & $r \cdot r / r \pm I r / \Lambda^{g}$ & $\wedge \varepsilon N \vee \vee r \Delta / \vee^{\mathrm{a}}$ & $F \Delta V / r \pm r r / \Lambda^{g}$ & $|r r| / \Delta \pm r \Delta / q^{D}$ & شيكيمات \\
\hline$\Lambda V / r \pm r / \gamma^{f}$ & $q N r \pm 1 / v^{f}$ & $1 \Delta q / q \pm \tau / \Lambda^{c d}$ & $\mid \Delta r / \backslash \pm \varepsilon / \gamma^{d}$ & 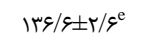 & $\mid \varepsilon q / q \pm \Delta / \mathrm{c}^{\mathrm{c}}$ & $\mid \Lambda \Delta / \Lambda \pm r / r^{b}$ & $r r v / \Lambda \pm r / v^{a}$ & سو كسينات \\
\hline
\end{tabular}

با اعمال تنش شورى اختلاف قندها در هر دو زمان بهطور

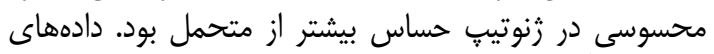

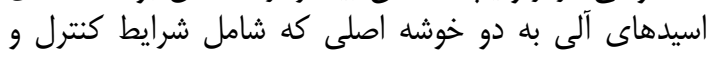

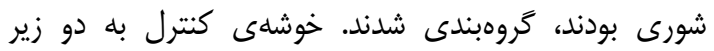

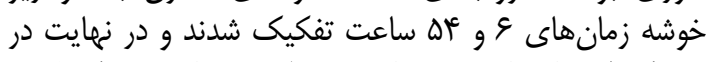

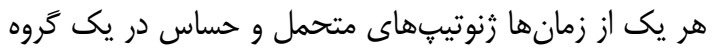

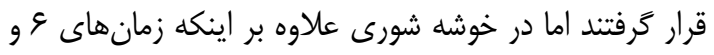

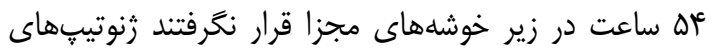

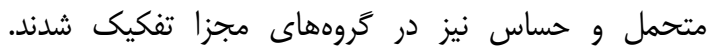

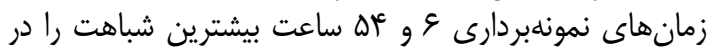

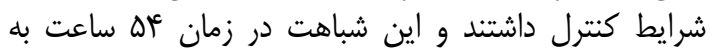

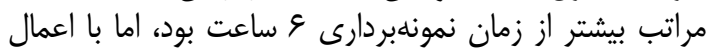

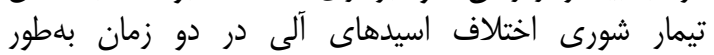
محسوسى كاهش يافت.
كرووبندى نمونهها بر اساس مقادير متابوليتها

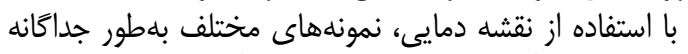

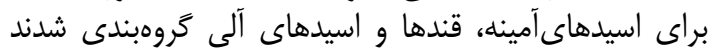

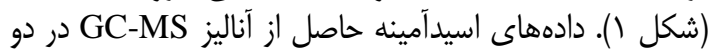

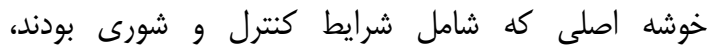

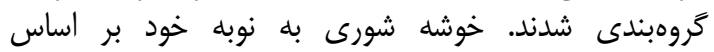

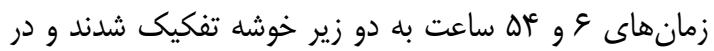

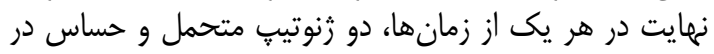

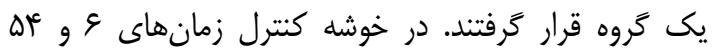

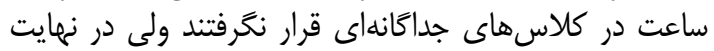

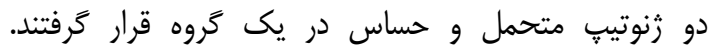

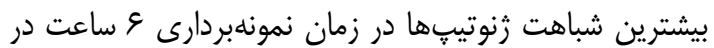

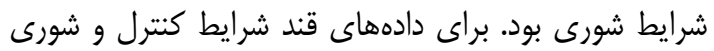

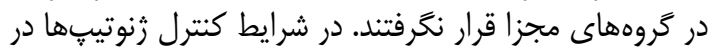

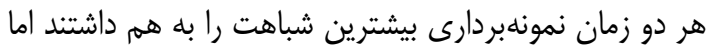




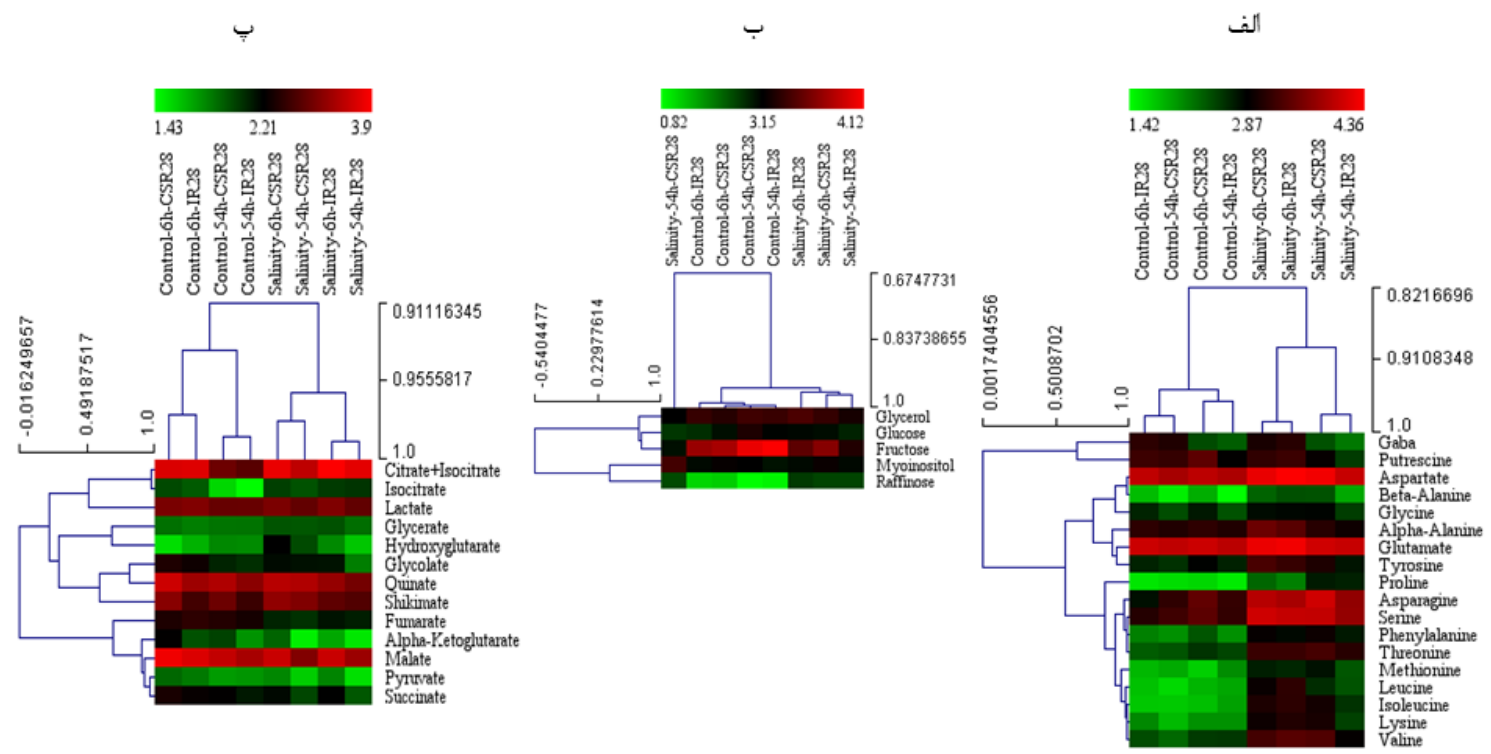

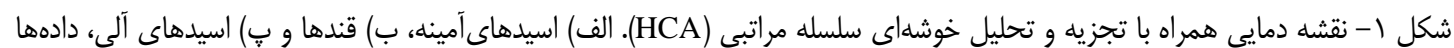

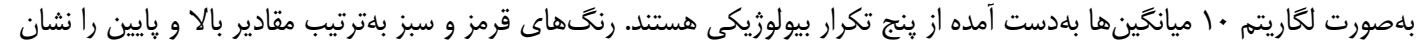
مىدهند

Figure 1. Heatmap combined with hierarchical cluster analysis (HCA). a) Amino acids, b) Sugar and C) Organic acids, the data are $\log _{10}$ of the means obtained of the five biological replications. The Red and green colors indicate the higher and the lower values, respectively

آنتىاكسيدانى يس آنهاز وقود.

$$
\text { تشكر و قدردانى }
$$

از موسسه بينالمللى تحقيقات برنج درانى در فيلييين (IRRI)

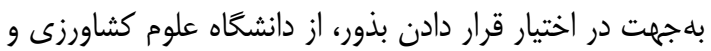

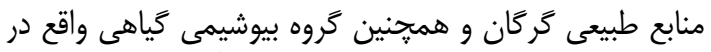

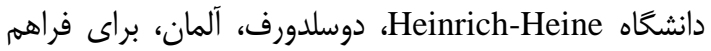

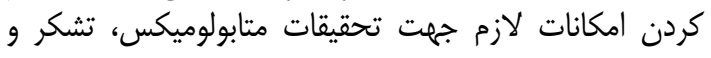

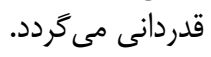

$$
\begin{aligned}
& \text { در اين تحقيق يروفايلينگ متابوليتها بر اساس آناليز } \\
& \text { GC-MS }
\end{aligned}
$$

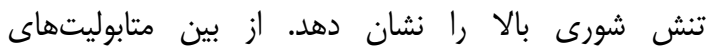

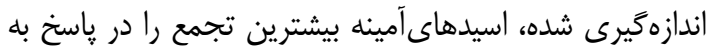

1. Ashraf, M. and M.R. Foolad. 2007. Role of glycine betaine and proline in improving plant abiotic stress resistance. Environmental and Experimental Botany, 59: 206-216.

2. Batista-Silva, W., B. Heinemann, N. Rugen, A. Nunes-Nesi, W.L. Araújo, H.P. Braun and T.M. Hildebrandt. 2019. The role of amino acid metabolism during abiotic stress release. Plant, Cell and Environment, 42(5): 1630-1644.

3. Chaves, M., J. Flexas and C. Pinheiro. 2009. Photosynthesis under drought and salt stress: regulation mechanisms from whole plant to cell. Annals of Botany, 103(4): 551-560.

4. Fiehn, O., J. Kopka, P. Dörmann, T. Altmann, R.N. Trethewey and L. Willmitzer. 2000. Metabolite profiling for plant functional genomics. Nature Biotechnology, 18(11): 1157.

5. Fiehn, O. 2002. Metabolomics - the link between genotypes and phenotypes. Plant Molecular Biology, 48: 155-171.

6. Food and Agriculture Organization of the United Nations, FAOSTAT. Rome, Italy. FAO. 2018. Available at: http://www.fao.org/faostat/en/\#data/QC (Accessed 01 Apr 2020).

7. Fougere, F., D.L. Rudulier and J.G. Streeter. 1991. Effects of salt stress on amino acids, organic acids, and carbohydrate composition of roots, bacteroids, and cytosol of alfalfa (Medicago sativa L.). Plant Physiology, 96: 1228-1236.

8. Funck, D., B. Stadelhofer and W. Koch. 2008. Ornithine- $\delta$-aminotransferase is essential for arginine catabolism but not for proline biosynthesis. BMC Plant Biology, 40: 1-14. 
9. Gerona, M.E.B., M.P. Deocampo, J.A. Egdane, A.M. Ismail and M.L. Dionisio-Sese. 2019. Physiological Responses of Contrasting Rice Genotypes to Salt Stress at Rice Reproductive Stage. Science, 26(4): 207-219.

10. Good, A.G. and S.T. Zaplachinski. 1994. The effects of drought stress on free amino acid accumulation and protein-synthesis in Brassica napus. Physioloy Plant, 90: 9-14.

11. Gu, J., K. Weber, E. Klemp, G. Winters, S.U. Franssen, I. Wienpahl and E. Bornberg Bauer. 2012. Identifying core features of adaptive metabolic mechanisms for chronic heat stress attenuation contributing to systems robustness. Integrative Biology, 4(5): 480-493.

12. Hashemi, A., GH. Nematzadeh, GH. Hosseini Salekdeh, S.A. Hosseini and M.R. Hajirezaei. 2012. Physiological and metabolic responses in rice under salt stress. Crop Biotechnology, 2: 1-14 (In Persian).

13. Heenan, D., L. Lewin and D. McCaffery. 1988. Salinity tolerance in rice varieties at different growth stages. Australian Journal of Experimental Agriculture, 28(3): 343-349.

14. Hu, C.A., A.J. Delauney and D.P. Verma. 1992. A bifunctional enzyme (delta 1-pyrroline-5carboxylate synthetase) catalyzes the first two steps in proline biosynthesis in plants. Proceedings of the National Academy of Sciences, 89(19): 9354-9358.

15. Hu, L., K. Zhou, Y. Li, X. Chen, B. Liu, C. Li. and F. Ma. 2018. Exogenous myo-inositol alleviates salinity-induced stress in Malus hupehensis Rehd. Plant Physiology and Biochemistry, 133: 116-126.

16. Jia, Q., D. Kong, Q. Li, S. Sun, J. Song, Y. Zhu and J. Huang. 2019. The function of inositol phosphatases in plant tolerance to abiotic stress. International Journal of Molecular Sciences, 20(16): 3999.

17. Jiménez-Bremont, J.F., A. Becerra-Flora, E. Hernández-Lucero, M. Rodríguez-Kessler, J.A. AcostaGallegos and J.G. Ramírez-Pimentel. 2006. Proline accumulation in two bean cultivars under salt stress and the effect of polyamines and ornithine. Biologia Plantarum, 50(4): 763-766.

18. Joshi, V., J.G. Joung, Z.J. Fei and G. Jander. 2010. Interdependence of threonine, methionine and isoleucine metabolism in plants: accumulation and transcriptional regulation under abiotic stress. Amino Acids, 39: 933-947.

19. Kim, J.K., T. Bamba, K. Harada, E. Fukusaki and A. Kobayashi. 2007. Time-course metabolic profiling in Arabidopsis thaliana cell cultures after salt stress treatment. Journal of Experimental Botany, 58: 415-424.

20. Lehmann, M., M. Laxa, L. Sweetlove, A. Fernie and T. Obata. 2012. Metabolic recovery of Arabidopsis thaliana roots following cessation of oxidative stress. Metabolomics, 8: 143-153.

21. Liang, J,. Y. Qu, C. Yang, X. Ma, G. Cao and Z. Zhao. 2010. Identification of QTLs associated with salt or alkaline tolerance at the seedling stage in rice under salt or alkaline stress. Euphytica, 01: 201.

22. Lisec, J., N. Schauer, J. Kopka, L. Willmitzer and A. Fernie. 2006. Gas chromatography mass spectrometry-based metabolite profiling in plants. Nature Protocols, 1: 387-396.

23. Nemati, I., F. Moradi, M.A. Esmaeili and S. Gholizadeh. 2011. The effect of salinity stress on ions and soluble sugars distribution in leaves, leaf sheaths and roots of rice (Oryza sativa L.) seedlings. Plant Soil Environ, 1: 26-33.

24. Nishizawa, A., Y. Yabuta and S. Shigeoka. 2008. Galactinol and raffinose constitute a novel function to protect plants from oxidative damage. Plant Physiology, 147(3): 1251-1263.

25. Omrani, B. and S. Moharramnejad. 2017. Study of salinity tolerance in four maize (Zea Mays L.) Hybrids at Seedling Stage. Journal of Crop Breeding, 9(24): 79-86 (In Persian).

26. Pandit, A., V. Rai, T.R. Sharma and N. K. Singh. 2011. Differentially expressed genes in sensitive and tolerant rice varieties in response to salt-stress. Plant Biochemistry and Biotechnology, 20: 149-154.

27. Valliyodan, B. and H.T. Nguyen. 2006. Understanding regulatory networks and engineering for enhanced drought tolerance in plants. Current Opinion in Plant Biology, 9: 1-7.

28. Verbruggen, N. and C. Hermans. 2008. Proline accumulation in plants: a review. Amino Acids, 35 : 753-759.

29. Wang, J., J. Zhu, Y. Zhang, F. Fan, W. Li, F. Wang and J. Yang. 2018. Comparative transcriptome analysis reveals molecular response to salinity stress of salt-tolerant and sensitive genotypes of indica rice at seedling stage. Scientific reports, 8(1): 1-13.

30. Wang, W.S., X.Q. Zhao, M. Li, L.Y. Huang, J.L. Xu, F. Zhang and Z.K. Li. 2016. Complex molecular mechanisms underlying seedling salt tolerance in rice revealed by comparative transcriptome and metabolomic profiling. Journal of Experimental Botany, 67(1): 405-419.

31. Widodo, P., J.H.E. Newbigin, M. Tester, A. Bacic and U. Roessner. 2009. Metabolic responses to salt stress of barley (Hordeum vulgare L.) cultivars, Sahara and Clipper, which differ in salt tolerance. Journal of Experimental Botany, 60: 4089-4103.

32. Yancey, P.H. 2005. Organic osmolytes as compatible, metabolic and counteracting cytoprotectants in high osmolarity and other stresses. Journal of Experimental Biology, 208(15): 2819-2830.

33. Yoshida, S., D.A. Forno and J.H. Cock. 1976. Laboratory manual for physiological studies of rice. $3^{\text {th }}$ edn. Los Banos, Laguna, Philippines, 83 pp.

34. Zhu, J.K. 2002. Salt and drought stress signal transduction in plants. Annual Review of Plant Biology, 53(1): 247-273. 


\title{
Response of some of Primary Metabolites in Rice (Oryza sativa L.) Root to Salinity Stress
}

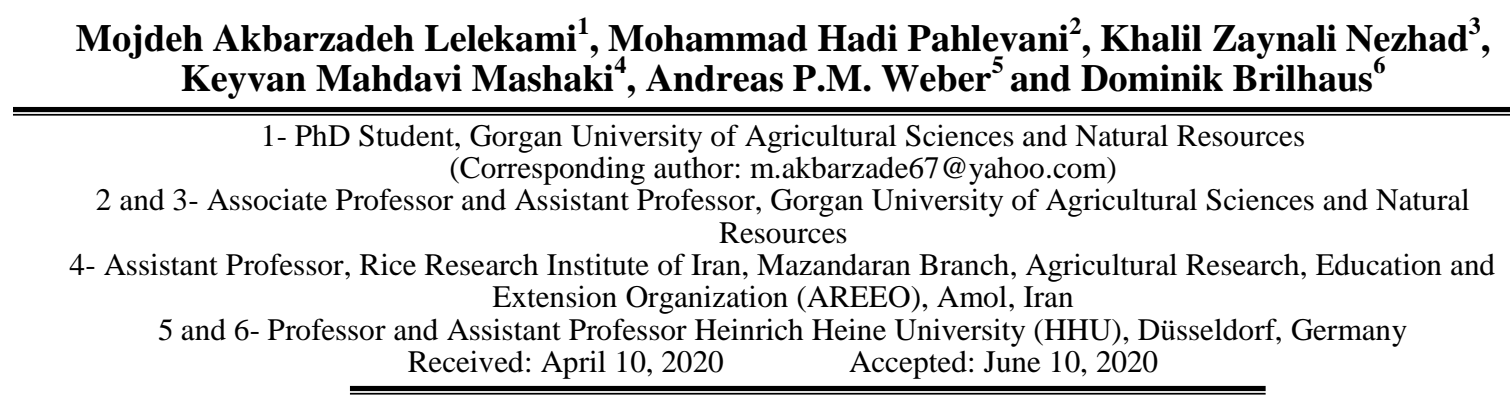

\begin{abstract}
In order to investigate the metabolic responses of rice root under salinity stress at seedling stage, an experiment was conducted at greenhouse with five replications as factorial in a completely randomized design including two salinity levels $(0$ and $150 \mathrm{mM})$, two sampling times (6h and 54h) and two genotypes CSR28 (tolerant) and IR28 (susceptible). In total, GCMS analysis identified 36 primary metabolites (including 18 amino acids, five sugars and sugar alcohols and 13 organic acids). The amino acids showed the highest accumulation in facing to high salinity. Moreover, the genotypes showed more differences after the long-term of salinity treatment and the tolerant genotype CSR28 had the highest accumulation of amino acids suggesting the role of osmotic adjustment mechanisms on inducing of salt-tolerance. Among the sugars, raffinose and myoinositol had more accumulation specifically in the tolerant genotype at $54 \mathrm{~h}$ time point, indicating their antioxidant activity. Generally, these findings emphasized the importance of identification and dissection of metabolic pathways for understanding of the salttolerance molecular mechanisms and consequently improving the development of salt-tolerant varieties in rice.
\end{abstract}

Keywords: Seedling stage, Amino acid, Sugar, Osmotic adjustment, GC-MS analysis 